\title{
Evolução da Morfologia de Fases de Blendas PA6/AES em Extrusora de Dupla Rosca e Moldagem por Injeção
}

\author{
Adriane Bassani, Elias Hage Jr., Luiz A. Pessan \\ Departamento de Engenharia de Materiais, UFSCar \\ Ana V. Machado, José A. Covas \\ Departamento de Engenharia de Polímeros, Universidade do Minho, Portugal
}

\begin{abstract}
Resumo: A evolução da morfologia de fases em blendas não reativas e reativas de poliamida- 6 com copolímero de acrilonitrila/EPDM/estireno (AES) em uma extrusora de rosca dupla co-rotacional foi investigada. A evolução da morfologia de fases ao longo da extrusora de rosca dupla foi monitorada através da coleta de pequenas amostras em válvulas no barril da extrusora e caracterização por microscopia eletrônica de transmissão (MET). Foram utilizados como compatibilizantes reativos os copolímeros metacrilato de metila-co-anidrido maleico (MMA-MA) e metacrilato de metila-co-metacrilato de glicidila (MMA-GMA). Os grupos anidrido maleico e epóxi nos copolímeros podem reagir com os grupos finais de cadeia da poliamida durante o processamento no estado fundido e melhorar a interação na interfase do sistema PA6/AES. A blenda PA6/AES não compatibilizada apresentou uma morfologia grosseira de fases onde a fase AES não está bem dispersa na matriz de PA6 devido à falta de interações adequadas entre os componentes da blenda. A adição do compatibilizante MMA-GMA não proporcionou boa dispersão de fases e não melhorou as propriedades mecânicas da blenda, provavelmente porque as possíveis reações no sistema são lentas e podem não ocorrer na extrusora. Por outro lado, a blenda compatibilizada com o copolímero MMA-MA apresentou uma excelente resistência ao impacto à temperatura ambiente e uma diminuição na temperatura de transição dúctil-frágil. As partículas de AES apresentam-se muito mais refinadas e sofrem uma redução significativa em seu tamanho já no início do processo de extrusão. A morfologia observada nas amostras injetadas apresentou uma boa correlação com as propriedades mecânicas obtidas.
\end{abstract}

Palavras-chave: Poliamida-6, Morfologia, Compatibilização reativa.

\section{Evolution of Phase Morphology of PA6/AES Blends During the Twin Screw Extrusion and Injection Molding Processes}

Abstract: The evolution of phase morphology in non-reactive vs reactive blends with polyamide and ethylene-propylenediene elastomer grafted with styrene-acrylonitrile copolymer (AES) in a co-rotating twin-screw extruder was investigated. The morphological evolution of these blends along a twin-screw extruder was monitored by quickly collecting small samples from the melt at specific extruder barrel locations and characterizing them with a transmission electron microscope (TEM). The copolymers methyl methacrylate-co-maleic anhydride (MMA-MA) and methyl methacrylate-co-glycidyl methacrylate (MMA-GMA) were used as compatibilizing agents. The maleic anhydride and the epoxy groups of the copolymers can react with the polyamide end groups during melt processing and improve the interphase interaction in the PA6/AES system. The uncompatibilized blends showed a coarse phase morphology where the AES phase is not well dispersed in the PA6 matrix due to lack of adequate interaction between the components. The addition of MMA-GMA compatibilizer neither promoted good phase dispersion or improved the mechanical properties of the blends, probably because the possible reactions are very slow and may not occur inside the extruder. On the other hand, the addition of the MMA-MA copolymer promotes better impact strength and good phase dispersion in the blend. The AES particles undergo significant reduction in the first stages of the mixture inside the extruder. The morphology observed for the injection molded specimens was correlated with the mechanical properties.

Keywords: Polyamide, reactive compatibilization, morphology.

\section{Introdução}

As poliamidas (PA) são extensivamente utilizadas em aplicações de engenharia devido a sua alta tenacidade sob certas condições e também excelente resistência química. Uma grande limitação para a aplicação da PA é sua fragili- dade sob impacto a baixas temperaturas. Para melhorar sua performance tem se utilizado a tenacificação da poliamida com materiais elastoméricos ${ }^{[1,2]}$. Blendas de poliamidas com copolímero AES (acrilonitrila-EPDM-estireno) têm vários atrativos, incluindo a redução da absorção de umidade, devido à diluição da poliamida com um material hidrofóbico.

Autor para correspondência: Luiz A. Pessan, Departamento de Engenharia de Materiais, UFSCar, Via Washington Luiz Km 235, CEP: 13.565-905, São Carlos, SP. E-mail: pessan@power.ufscar.br 
Características do elastômero EPDM, como, baixa temperatura de transição vítrea, excelente estabilidade térmica e UV, o tornam um candidato ideal para a modificação do impacto de certos polímeros ${ }^{[3-6]}$.

A performance das blendas poliméricas imiscíveis é determinada, principalmente, pela morfologia e dispersão das fases, o que tem sido objeto de estudo de muitas pesquisas. A morfologia final de blendas poliméricas é uma conseqüência de interações complexas entre os componentes ao longo de toda a extrusora, desde a sua alimentação até a saída da matriz da extrusora. Estas interações estão relacionadas com diferentes tipos de variáveis, entre elas, características reológicas e físicas dos componentes (razão de viscosidade, razão de elasticidade, tensão interfacial, temperatura de transição vítrea, temperatura de fusão, etc.); composição das blendas; compatibilização (tipo, concentração, peso molecular dos grupos funcionais); intensidade de mistura (tipo de extrusora, configuração da rosca, condições de processamento, etc.). Portanto, é de extrema importância a compreensão da evolução da morfologia de fases das blendas durante o processamento e sua correlação com as condições de processamento utilizadas ${ }^{[7-17]}$

A maioria das pesquisas sobre o desenvolvimento de morfologia de fases em blendas poliméricas durante o processamento concentra-se no estudo de blendas mecânicas ou não compatibilizadas. Alguns autores têm observado que o desenvolvimento da morfologia de blendas mecânicas é um processo contínuo ao longo de toda a extrusora, enquanto que para blendas compatibilizadas a morfologia se desenvolve muito mais rapidamente e o tamanho final das partículas encontra-se mais finamente disperso ${ }^{[11]}$.

Extrusoras de dupla rosca co-rotacional interpenetrantes têm sido muitas vezes utilizadas para preparar blendas e/ou modificar a estrutura de polímeros em escala comercial. Entretanto, poucas informações estão disponíveis sobre o desenvolvimento da morfologia ou sobre a evolução da conversão química dentro deste tipo de equipamento. Uma das razões para esta situação reside na dificuldade de coleta de amostras durante o processamento, o que vem sendo estudado e melhorado recentemente por alguns pesquisadores. O principal objetivo é produzir informações em tempo real, as quais poderão ser utilizadas para melhorar a eficiência dos processos químicos e/ou físicos, o projeto de equipamentos, e a capacidade de controle on-line dos pro$\operatorname{cessos}^{[14]}$.

Em um trabalho recente, foi desenvolvido um dispositivo para coleta de amostras em menos de $5 \mathrm{~s}^{[14]}$. Este dispositivo pode ser inserido entre os segmentos do barril da extrusora, possibilitando a obtenção de amostras em espaços relativamente pequenos ao longo do comprimento da extrusora.

Neste trabalho, foi utilizado este novo dispositivo de coleta de amostras ao longo da extrusora para análise da evolução da morfologia de fases de blendas compatibilizadas e não compatibilizadas baseadas em PA6 e AES. Analisou-se também a morfologia de fases de corpos-de-prova injetados e sua correlação com as propriedades mecânicas sob impacto e tração. Os copolímeros metacrilato de metila-co-anidrido maleico (MMA-MA) e metacrilato de metila-co-metacrilato de glicidila (MMA-GMA) foram utilizados como agentes de compatibilização.

\section{Materiais e Métodos}

\section{Materiais}

A Tabela 1 apresenta as características dos materiais utilizados neste trabalho.

A poliamida-6 utilizada foi um material disponível comercialmente (Ultramid B3), fornecida pela BASF. O copolímero de acrilonitrila-EPDM-estireno (AES) utilizado foi o Royaltuf 372P20, fornecido pela Uniroyal Chemical. O copolímero utilizado como agente de compatibilização foi o metacrilato de metila-co-anidrido maleico (MMA-MA), contendo 1,3\% em peso de MA, sintetizado em nossos laboratórios de acordo com o procedimento descrito na literatura ${ }^{[18]}$. O outro compatibilizante utilizado foi o metacrilato de metilaco-metacrilato de glicidila (MMA-GMA), contendo $10 \%$ em peso de GMA, também sintetizado em nossos laboratórios de acordo com o procedimento descrito na literatura ${ }^{[19]}$.

Tabela 1. Características dos materiais utilizados

\begin{tabular}{|c|c|c|c|c|}
\hline Polímero & PA6 & AES & MMA-MA1 & MMA-GMA10 \\
\hline Fornecedor & BASF & Uniroyal Chemical & Polimerização em Solução & Polimerização em Massa \\
\hline Composição & $\begin{array}{c}{\left[\mathrm{NH}_{2}\right]^{\mathrm{a}}=35,3 \mu \mathrm{eq} / \mathrm{g}} \\
{[\mathrm{COOH}]^{\mathrm{a}}=53,6 \mu \mathrm{eq} / \mathrm{g}}\end{array}$ & $\begin{array}{l}50 \% \mathrm{EPDM} \\
50 \% \mathrm{SAN} \\
\left(24 \% \mathrm{AN}^{[4]}\right.\end{array}$ & $1,3 \%$ em peso $\mathrm{MA}^{\mathrm{d}}$ & $10 \%$ em peso $\mathrm{GMA}^{\mathrm{d}}$ \\
\hline $\bar{M}_{n}(\mathrm{~g} / \mathrm{mol})$ & $16.100^{b}$ & - & $18.800^{\mathrm{e}}$ & $22.600^{\mathrm{e}}$ \\
\hline Viscosidade relativa $^{c}$ & 1 & 5,8 & 0,3 & 1 \\
\hline $\begin{array}{l}\text { nteúdo dos grupos finais } \\
\text { tido pela media dos grup } \\
\text { rque após } 10 \text { min de mist } \\
\text { onteúdo de MA obtido po } \\
\text { btido por GPC }\end{array}$ & $\begin{array}{l}\text { los por titulação } \\
\text { nais } \\
220^{\circ} \mathrm{C} \text { e } 60 \mathrm{rpm} \text {, relativo a } \\
\text { lação (\% em peso) }\end{array}$ & A6 & & \\
\hline
\end{tabular}




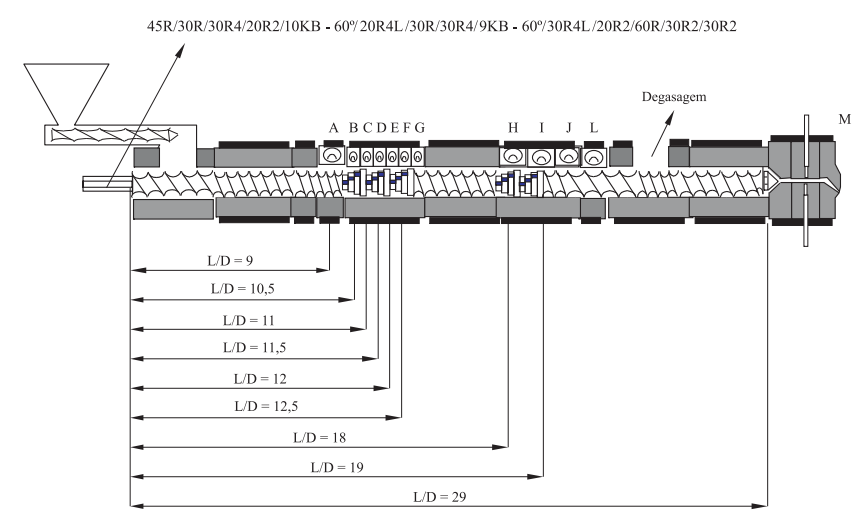

Figura 1. Configuração da rosca e localização dos coletores.

\section{Preparação das blendas}

As blendas foram preparadas em uma extrusora de rosca dupla co-rotacional interpenetrante modular Leistritz LSM 30.34 acoplada com um acessório para coleta de amostra durante o processamento. Anterior a cada processamento todos os materiais foram secos a vácuo por 24 horas a $80^{\circ} \mathrm{C}$.

A configuração da rosca $(L / D=29)$ e a localização dos coletores de amostra (A, B, C, D, E, F, G, H, I, J, L, M) estão apresentados na Figura 1. Após a coleta da amostra da extrusora esta foi imediatamente resfriada em nitrogênio líquido para congelar a morfologia presente e garantir idêntica história térmica para todas as amostras.

As blendas binárias (PA6/AES) foram preparadas na composição de 70/30 (\% em peso) e a blendas compatibilizadas com MMA-MA1 (PA6/AES/MMA-MA1) e com MMAGMA10 (PA6/AES/MMA-GMA10) foram preparadas na composição $66,5 / 28,5 / 5$ (\% em peso). Para a preparação das misturas todos os materiais foram alimentados em uma única etapa na extrusora. O processo de extrusão foi realizado com perfil de temperatura de $220^{\circ} \mathrm{C}$, vazão de alimentação de 4 $\mathrm{Kg} / \mathrm{h}$ e rotação da rosca de $150 \mathrm{rpm}$.

\section{Caracterização Mecânica e Morfológica}

Os efeitos da compatibilização reativa foram avaliados através de propriedades mecânicas e evolução da morfologia. As amostras para testes de resistência ao impacto e tração foram preparadas por moldagem por injeção (Arburg Allrounder 270 V) utilizando temperatura de injeção de $220-250{ }^{\circ} \mathrm{C}$ e temperatura do molde de $60^{\circ} \mathrm{C}$. Os ensaios de tração foram realizados segundo a norma ASTM D638, utilizando uma máquina de ensaio universal Instron, modelo 5569, a uma velocidade de $5 \mathrm{~mm} / \mathrm{min}$. Os ensaios de impacto sob entalhe tipo IZOD foram realizados segundo a norma ASTM D256, em um equipamento CEAST modelo RESIL 25, equipado com câmera para aquecimento e resfriamento dos corpos-de-prova. Anterior aos testes mecânicos, todas as amostras foram condicionadas a temperatura ambiente em dessecador por no mínimo 48 horas.

A morfologia foi analisada por microscopia eletrônica de transmissão (MET) utilizando um microscópio Phillips operando a $120 \mathrm{kV}$. As amostras foram obtidas das diferentes localizações ao longo da extrusora e de corpos-de-prova de impacto moldados por injeção, perpendiculares à direção do fluxo, na região do entalhe. Estas amostras foram submetidas ao trimming a temperatura ambiente e logo após microtomadas criogenicamente $\left(-60^{\circ} \mathrm{C}\right)$ em secções ultrafinas $(30 \mathrm{~nm})$. Para a observação no MET, as secções microtomadas foram expostas ao vapor de de tetróxido de Ósmio $\left(\mathrm{OsO}_{4}\right)$ em solução aquosa (1\%) por 15 horas.

\section{Evidências da reatividade in situ}

A reatividade dos sistemas (PA6/MMA-MA1) e (PA6/ MMA-GMA10) foi avaliada através de testes de reometria de torque (Haake Rheomix - 600), utilizando uma rotação de 60 $\mathrm{rpm}$, temperatura de mistura de $220^{\circ} \mathrm{C}$, e tempo de 15 minutos de mistura. $\mathrm{O}$ aumento no torque com o tempo de mistura é um indicativo da ocorrência de reações químicas entre os grupos funcionais dos componentes, isto é, entre os grupos anidrido maleico do MMA-MA com os grupos finais de cadeia amina da poliamida-6. E também as reações dos grupos epóxi do MMA-GMA com os grupos finais de cadeia, tanto aminas como ácidos carboxílicos da poliamida- 6 .

\section{Resultados e Discussão}

\section{Propriedades Mecânicas}

Através da Tabela 2, observa-se que para a blenda binária PA6/AES não ocorre nenhuma alteração na resistência ao impacto em relação à poliamida-6 pura. A resistência à tração apresentou uma pequena redução com a adição do AES na matriz PA6. As condições de processamento não afetam

Tabela 2. Propriedades mecânicas para os materiais puros e para as blendas estudadas

\begin{tabular}{lccccc}
\hline \multicolumn{1}{c}{ Componentes } & $\begin{array}{c}\text { Resistência ao } \\
\text { Impacto IZOD } \\
(\mathbf{J} / \mathbf{m})\end{array}$ & $\begin{array}{c}\text { Módulo de } \\
\text { Elasticidade } \\
(\mathbf{M P a})\end{array}$ & $\begin{array}{c}\text { Tensão no } \\
\text { Escoamento } \\
(\mathbf{M P a})\end{array}$ & $\begin{array}{c}\text { Deformação na } \\
\text { Ruptura } \\
(\boldsymbol{\%})\end{array}$ & $\begin{array}{c}\mathbf{T}_{\text {DF }} \text { * } \\
\left({ }^{\circ} \mathbf{C}\right)\end{array}$ \\
\hline PA6 & $58 \pm 3$ & $2.900 \pm 63$ & $73 \pm 1$ & $52 \pm 8$ & $64 \pm 2$ \\
PA6/AES (70/30) & $58 \pm 6$ & $1.720 \pm 104$ & $36 \pm 0,8$ & $18 \pm 1$ & $71 \pm 2$ \\
PA6/AES/MMA-MA1 (66,5/28,5/5) & $227 \pm 3$ & $1.942 \pm 56$ & $44 \pm 1$ & $90 \pm 5$ & $29 \pm 0,6$ \\
PA6/AES/MMA-GMA10 (66,5/28,5/5) & $70 \pm 8$ & $1.877 \pm 72$ & $40 \pm 0,4$ & $19 \pm 1$ & $64 \pm 2$ \\
AES & $421 \pm 7$ & $590 \pm 24$ & $9,5 \pm 0,1$ & $59 \pm 4$ & $-55 \pm 2$ \\
\hline
\end{tabular}

$* \mathrm{~T}_{\mathrm{DF}}=$ Temperatura de transição dútil-frágil 


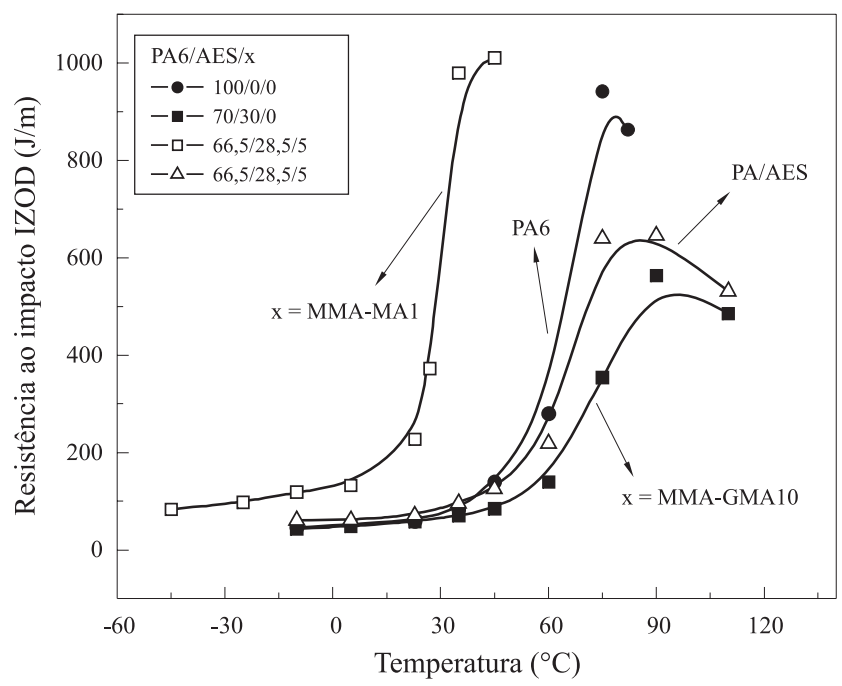

Figura 2 - Efeitos da temperatura sobre a resistência ao impacto IZOD para a PA6 pura; para as blendas PA6/AES (70/30); PA6/AES/MMA-MA1 e PA6/AES/MMA-GMA10 (66,5/28,5/5).

significativamente a resistência ao impacto e a tração do sistema binário PA6/AES ${ }^{[20]}$.

A Figura 2 apresenta a resistência ao impacto em função da temperatura para a PA6 pura, para as blendas compatibilizadas com $5 \%$ em peso do copolímero MMA-MA1 ou do copolímero MMA-GMA10 e para a blenda não compatibilizada. A temperatura de transição dútil-frágil $\left(\mathrm{T}_{\mathrm{DF}}\right)$ para a poliamida-6 e para a blenda PA6/AES não compatibilizada é de aproximadamente $70^{\circ} \mathrm{C}$ e $80^{\circ} \mathrm{C}$, respectivamente. Foi observado que a adição do AES não melhorou o comportamento de impacto para as blendas PA6/AES, uma vez que a resistência ao impacto à temperatura ambiente permaneceu muito semelhante a da PA6 pura e a temperatura de transição dúctil-frágil mudou para temperaturas mais elevadas. O sistema PA6/AES é incompatível, devido à falta de interações adequadas entre o AES e a PA6. Isto reforça a importância da adição de um agente compatibilizante para este sistema.

Com a adição dos compatibilizantes ao sistema, é esperado que os grupos anidrido maleico (MA) do copolímero MMA-MA e os grupos epóxi (GMA) do copolímero MMAGMA possam reagir durante o processamento com os grupos finais da poliamida- $6^{[3,11,13,14,17-20]}$ e o segmento de cadeia metacrilato de metila (MMA) destes copolímeros apresentem miscibilidade com a fase SAN do AES ${ }^{[21]}$.

Foi observado que a adição do compatibilizante MMAGMA10 ao sistema PA6/AES também tende a piorar as propriedades mecânicas deste sistema, pois a $\mathrm{T}_{\mathrm{DF}}$ foi deslocada para temperaturas superiores e a resistência ao impacto à temperatura ambiente sofreu pequenas alterações. Entretanto, a adição do copolímero MMA-MA1 tem um efeito positivo sobre a resistência ao impacto e propriedades de tração. A adição de somente $5 \%$ em peso do compatibilizante MMAMA1 aumentou a resistência ao impacto à temperatura ambiente e reduziu a temperatura de transição dútil-frágil em aproximadamente $40{ }^{\circ} \mathrm{C}$, como pode ser verificado na Figura 2 e na Tabela 2 . O efeito positivo da compatibilização também pode ser verificado nas propriedades de tração, onde ocorreu um aumento no módulo de elasticidade, na resistência a tração e na deformação na ruptura para a blenda PA6/ AES/MMA-MA1 em relação à blenda PA6/AES, como apresentado na Tabela 2. Estes resultados são comparáveis aos obtidos por Majumdar ${ }^{[22]}$ utilizando o compatibilizante IA (copolímero acrílico imidizado) em um sistema similar, PA6/ ABS. Kudva ${ }^{[19]}$ também obteve resultados semelhantes utilizando o compatibilizante MMA-GMA para as blendas PA6/ ABS, porém somente em condições específicas de intenso cisalhamento proporcionado pelas extrusoras de rosca dupla. Este comportamento foi relacionado ao fato de ambos os grupos finais de cadeia da poliamida, tanto aminas como ácidos carboxílicos, serem capazes de reagir com o grupamento epóxi do copolímero MMA-GMA.

\section{Reatividade dos sistemas}

Para melhor entender a evolução morfológica das blendas é importante verificar a reatividade dos compatibilizantes com os grupos finais de cadeia da poliamida-6. A reometria de torque foi utilizada para obter indicativos da ocorrência das possíveis reações químicas entre os componentes PA6, MMAMA1 e MMA-GMA10.

A Figura 3 apresenta as curvas de torque para a PA6, MMA-MA1 e para o sistema PA6/MMA-MA1, onde pode ser visto um aumento significativo no torque de mistura devido à adição do copolímero MMA-MA1 à PA6. Este fenômeno indica a ocorrência de reações químicas entre o MA e os grupos finais de cadeia da PA6 e, conseqüentemente, a formação de moléculas de copolímero PA6-g-MMA-MA, que podem ser localizados na interafase entre os componentes. A Figura 4 ilustra as possíveis reações entre o grupo anidrido maleico e o grupo final amina da poliamida ${ }^{[22]}$. É importante também observar o fato de que a taxa de reação entre o anidrido maleico e o grupo amida é mais rápida que a taxa de reação entre o GMA e o grupo amida, pois o aumento significativo no torque ocorre para tempos menores ${ }^{[23]}$.

A Figura 5 apresenta as curvas de torque em função do tempo de mistura para a PA6, MMA-GMA10 e para os sistemas PA6/MMA-GMA10. Pode-se observar que o nível de

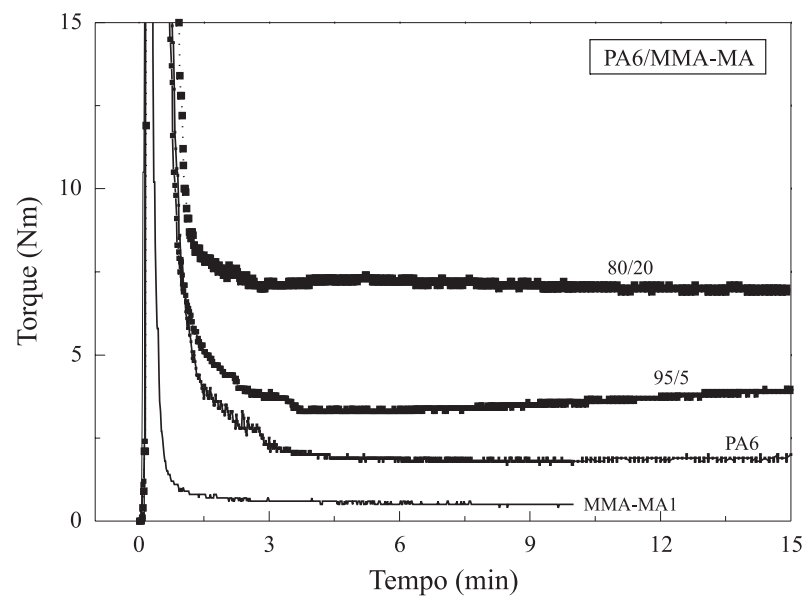

Figura 3. Torque versus tempo para a mistura PA6/MMA-MA1 (1,3\% em peso MA) a $220^{\circ} \mathrm{C}$ e $60 \mathrm{rpm}$. 


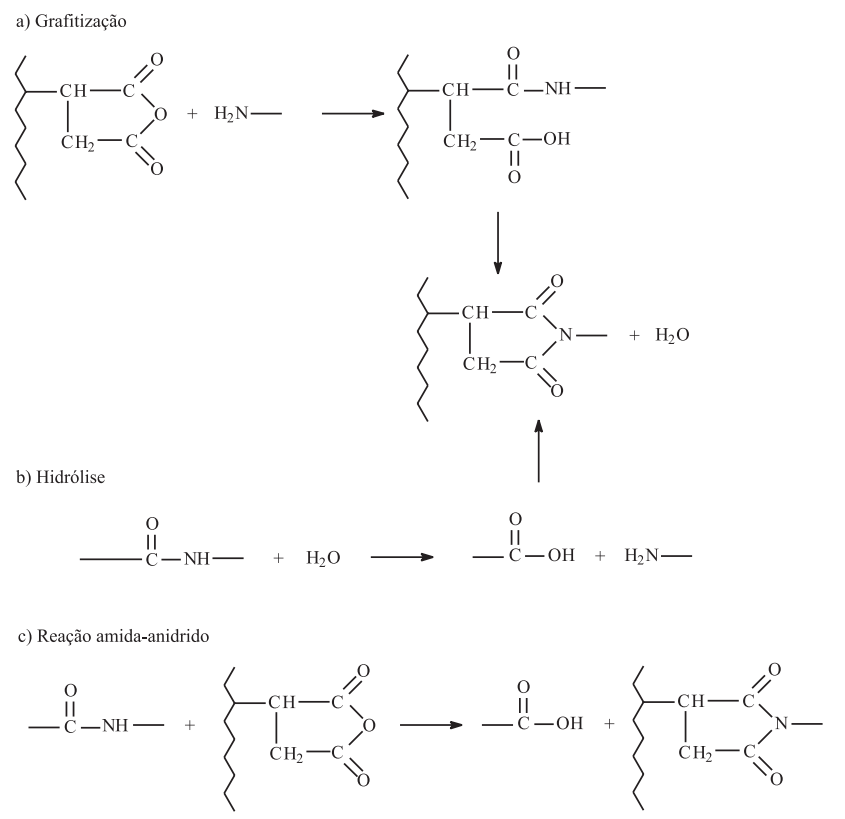

Figura 4. Principais reações que podem ocorrer em blendas de PA com polímeros funcionalizados com anidrido maleico ${ }^{[22]}$.

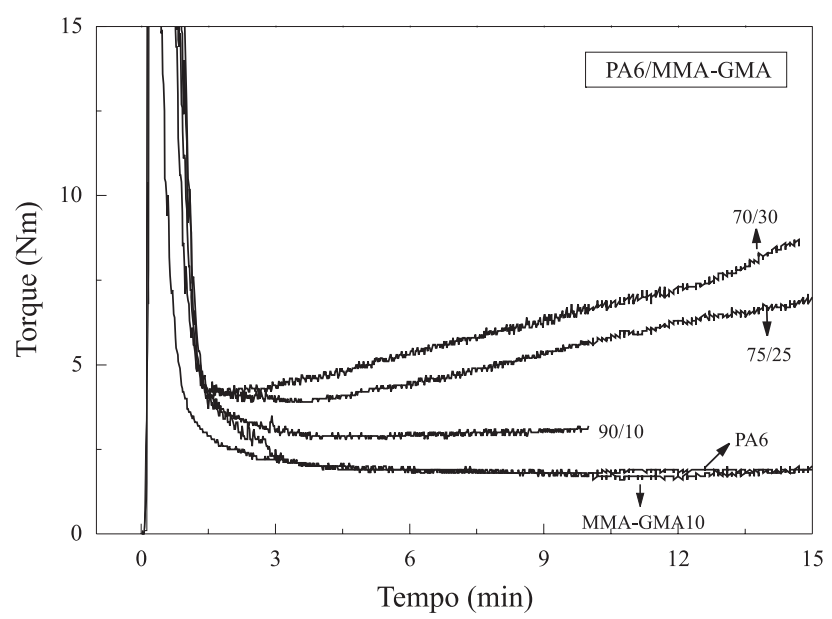

Figura 5. Torque versus tempo para a mistura PA6/MMA-GMA10 (10\% em peso GMA) a $220^{\circ} \mathrm{C}$ e $60 \mathrm{rpm}$.

torque para o sistema PA6/MMA-GMA10 (70/30) a 15 minutos de mistura é superior ao torque para o sistema PA6/MMAGMA10 (75/25) e que em ambos os sistemas a curva de torque apresenta um aumento contínuo com o tempo. A Figura 6 apresenta as possíveis reações entre os grupos finais da cadeia da poliamida e os grupos epóxi ${ }^{[19]}$. Os grupos amina e ácidos carboxílicos são ambos reativos e têm cinéticas de reação similares em relação ao grupamento epóxi do GMA no copolímero MMA-GMA, podendo com isso resultar em estruturas com ligações cruzadas. A ocorrência de ligações cruzadas pode ser a responsável pelo aumento do torque com o tempo de mistura para o sistema PA6/MMA-GMA10. Entretanto, isto não foi observado para o sistema PA6/MMA-MA1, pois o grupo anidrido maleico do copolímero MMA-MA é capaz de reagir somente com os grupos amina da poliamida.

Kudva e outros ${ }^{[19]}$ sugeriram que reações do tipo ligações cruzadas podem ocorrer devido à natureza bifuncional da

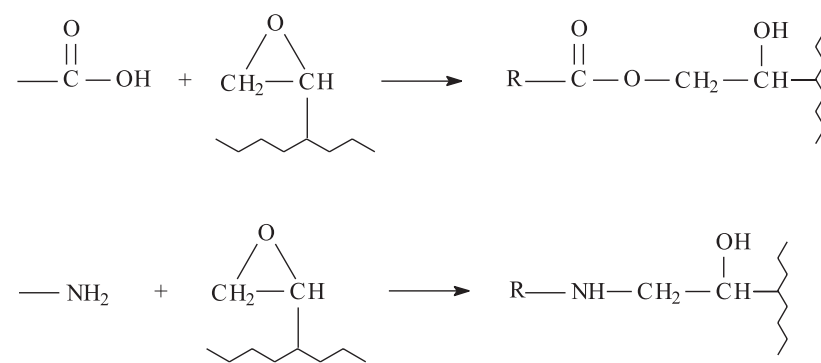

Figura 6 - Possíveis reações entre o grupo epóxi do copolímero MMAGMA10 e os grupos finais de cadeia da poliamida ${ }^{[19]}$.

poliamida em relação aos grupos epóxi do MMA-GMA. Isto faz com que as moléculas de PA6 atuem como uma ponte entre as partículas dispersas de ABS, dificultando assim a sua dispersão. A dispersão insatisfatória das partículas de ABS pode ser a responsável pelas baixas propriedades observadas nas blendas PA6/ABS/MMA-GMA.

A compatibilizarão interfacial através da adição e/ou formação in situ de copolímeros em bloco e/ou graftizados tem sido utilizada para controlar a morfologia das fases em blendas poliméricas. Este processo reduz a tensão interfacial entre as fases imiscíveis e geralmente produzem a redução no tamanho das partículas dispersas. Além disso, a presença deste copolímero em bloco ou graftizados na interface aumenta a região interfacial pela penetração das cadeias do copolímero dentro das fases adjacentes e com isso melhoram a adesão interfacial, reduzindo conseqüentemente a possibilidade de fratura interfacial. Tão importante quanto a redução da tensão interfacial é a estabilização da morfologia das fases contra a coalescência durante os processos de mistura.

\section{Evolução da morfologia de fases das blendas}

As Figuras 7, 8 e 9 apresentam a evolução morfológica durante o processamento das blendas PA6/AES (70/30), PA6/ AES/MMA-GMA10 (66,5/28,5/5) e PA6/AES/MMA-MA1 $(66,5 / 28,5 / 5)$, respectivamente. Nestas figuras as regiões escuras representam as partículas de AES e as regiões mais claras a matriz de poliamida-6.

A Figura 7 apresenta as fotomicrografias obtidas por MET para as blendas binárias PA6/AES obtidas dos diferentes pontos ao longo da rosca da extrusora, na saída da extrusora e dos corpos de prova injetados. A blenda não compatibilizada apresentou uma morfologia co-contínua das fases desde o início até a saída da extrusora com uma tendência a coalescência das fases, como pode ser visto nas Figuras $7 \mathrm{a}-7 \mathrm{~g}$. De modo geral, não foram observadas mudanças significativas no tamanho das partículas de AES dispersas ao longo de todo o comprimento da extrusora. No primeiro bloco de malaxagem (kneading blocks) foi observada uma morfologia co-contínua onde as partículas de AES aparecem interconectadas bem como a matriz PA6. No segundo bloco de malaxagem foi ainda observada uma morfologia co-contínua, porém pode ter ocorrido coalescência das partículas de AES.

A morfologia final de uma blenda polimérica imiscível é o resultado do balanço entre a ruptura e a coalescência das partículas dispersas induzidas pelo fluxo durante o processo de 

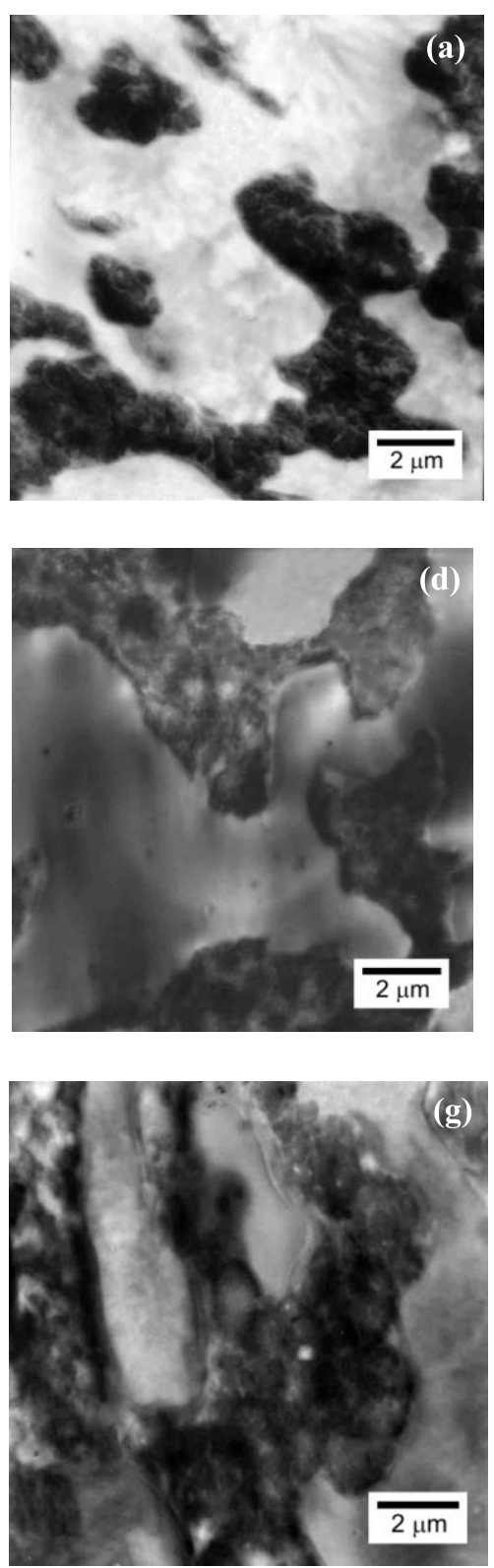
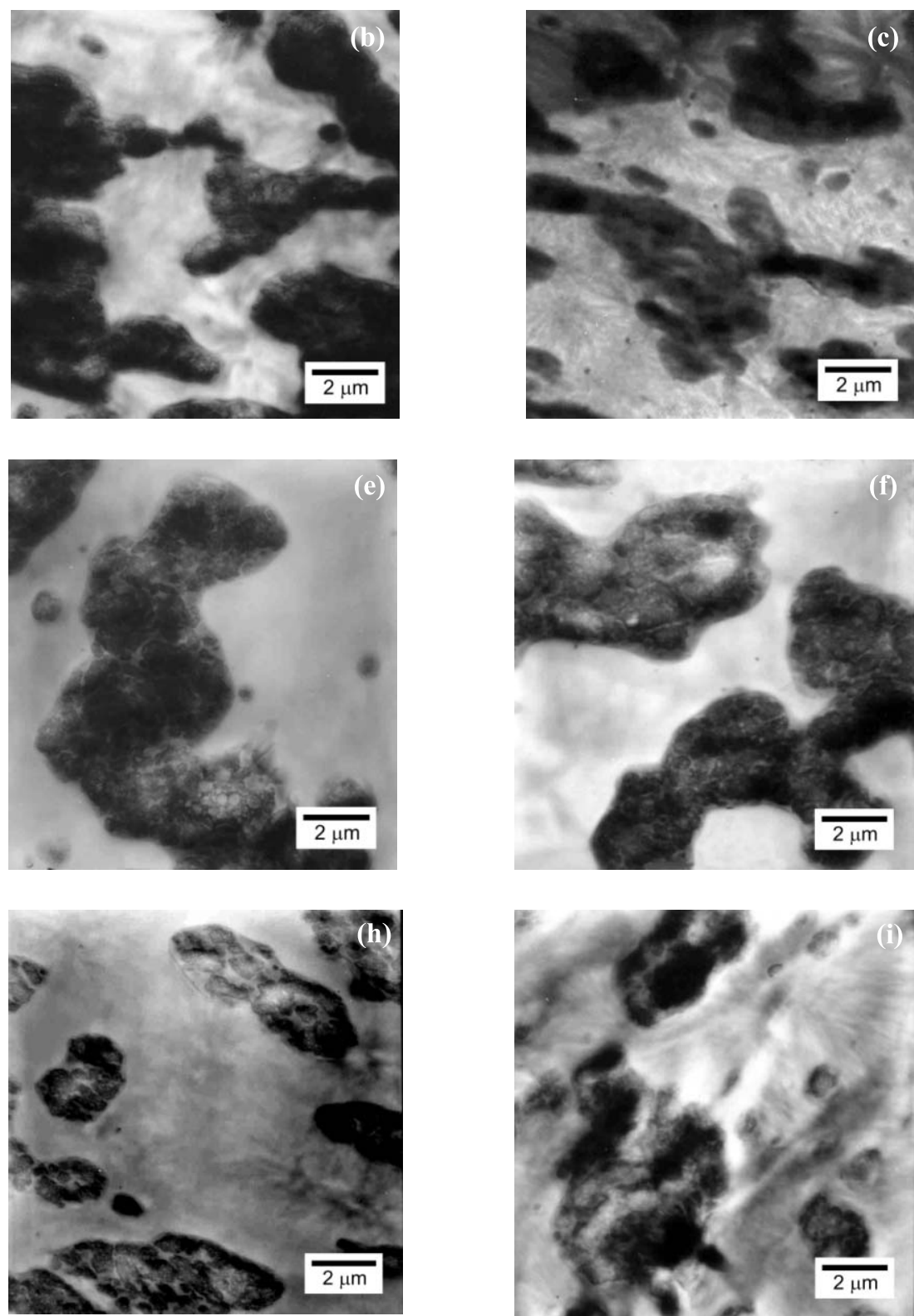

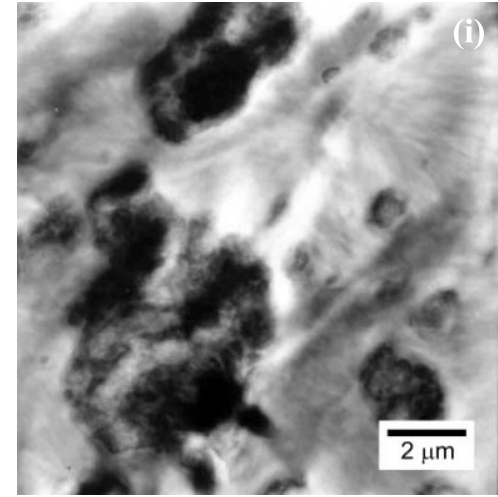

Figure 7 - Fotomicrografias das blendas PA6/AES (70/30) coletadas em diferentes posições na extrusora: (a) válvula B; (b) válvula C; (c) válvula D; (d) válvula $\mathrm{E}$; (e) válvula F; (f) válvula $\mathrm{H}$; (g) válvula I; (h) saída da extrusora $\mathrm{M}$ e (i) corpo de prova injetado.

mistura. A blenda imiscível PA6/AES (70/30) apresenta-se instável durante o processamento e o processo de coalescência está sobrepondo o processo de ruptura das partículas, pois o AES mostra-se mais grosseiramente disperso na matriz de PA6 após a passagem pelos blocos de malaxagem. $\mathrm{O}$ fenômeno de coalescência pode estar relacionado a muitas variáveis incluindo taxa de alimentação, rotação da rosca, geometria da rosca, concentração da fase dispersa, taxa das reações ao longo da extrusora e disponibilidade dos grupos funcionais ${ }^{[8]}$. A introdução de elementos de mistura na forma de blocos de malaxagem pode alterar o balanço entre a ruptura e a coalescência das partículas, sendo que a coalescência pode ser favorecida devido à maior probabilidade de colisão das partículas dispersas quando a blenda for submetida a uma mistura mais intensa. Por outro lado, na saída da extrusora (Figura 7h) foi observada uma morfologia de fases onde o AES apresentase disperso na matriz de PA6. Este fenômeno pode estar relacionado a capacidade da matriz da extrusora em proporcionar a ruptura das partículas devido à tensão elongacional desenvolvida na entrada da matriz. Várias investigações têm sido feitas para monitorar a evolução da morfologia de fases durante a extrusão ${ }^{[8-17]}$ e em geral têm-se observado uma redução contínua no tamanho das partículas ao longo da extrusora para blendas não compatibilizadas, o que é atribuído à ação de elementos de mistura ${ }^{[11]}$. Entretanto, observou-se também aumento no tamanho das partículas durante o processamento por extrusão usando perfis de rosca com a presença de elementos de mistura ${ }^{[8]}$.

Na morfologia final da blenda PA6/AES após injeção, as partículas de AES apresentam-se grosseiramente dispersas, 

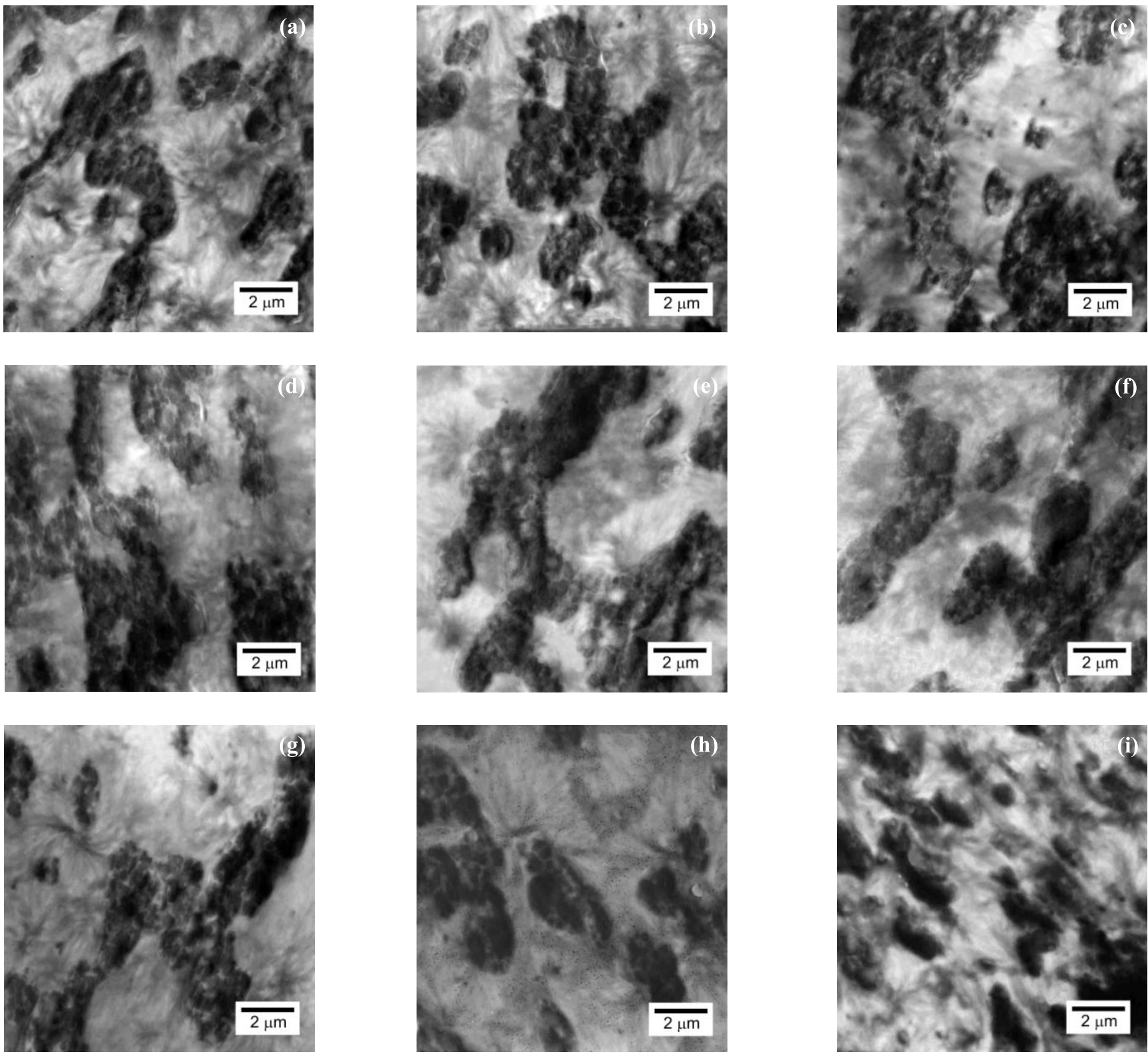

Figure 8 - Fotomicrografias das blendas PA6/AES/MMA-GMA10 (66,5/28,5/5) coletadas em diferentes posições na extrusora: (a) válvula B; (b) válvula $\mathrm{C}$; (c) válvula $\mathrm{D}$; (d) válvula $\mathrm{E}$; (e) válvula F; (f) válvula $\mathrm{H}$; (g) válvula I; (h) saída da extrusora $\mathrm{M}$ e (i) corpo de prova injetado.

o que se correlaciona com as baixas propriedades mecânicas.

Em blendas compatibilizadas com MMA-GMA10 as partículas também apresentam uma tendência a co-continuidade, porém o fenômeno de coalescência é menos pronunciado que em blendas não compatibilizadas, como pode ser verificado na Figura 8. Na morfologia final da blenda, o AES apresenta-se também grosseiramente disperso, resultando em baixas propriedades mecânicas. A Figura 8 mostra que o copolímero MMA-GMA não atua de forma eficiente como compatibilizante do sistema PA6/AES.

A Figura 9 apresenta as fotomicrografias obtidas por MET para as blendas ternárias PA6/AES/MMA-MA1 obtidas dos diferentes pontos ao longo da rosca da extrusora, na saída da extrusora e dos corpos de prova injetados. Para estas blendas a morfologia apresenta-se muito mais refinada em comparação com as Figuras 7 e 8. No início da extrusora, logo após a fusão dos componentes, ocorre uma diminuição bastante significativa no tamanho das partículas de AES dispersas, porém estas partículas têm uma tendência a coalescer com o decorrer do processamento. O fenômeno de coalescência nesta blenda é muito menos pronunciado que em blendas não compatibilizadas (PA6/AES) ou compatibilizadas com MMA-GMA10 na mesma localização na extrusora. É possível verificar claramente que nas localizações $\mathrm{B}$ e $\mathrm{C}$ as partículas de $\mathrm{AES}$ apresentam uma melhora significativa em sua dispersão nas misturas com a adição de compatibilizante MMA-MA1.

Em blendas compatibilizadas, tanto com MMA-GMA como com MMA-MA, foi possível também verificar a influência da matriz da extrusora em promover a ruptura das par- 

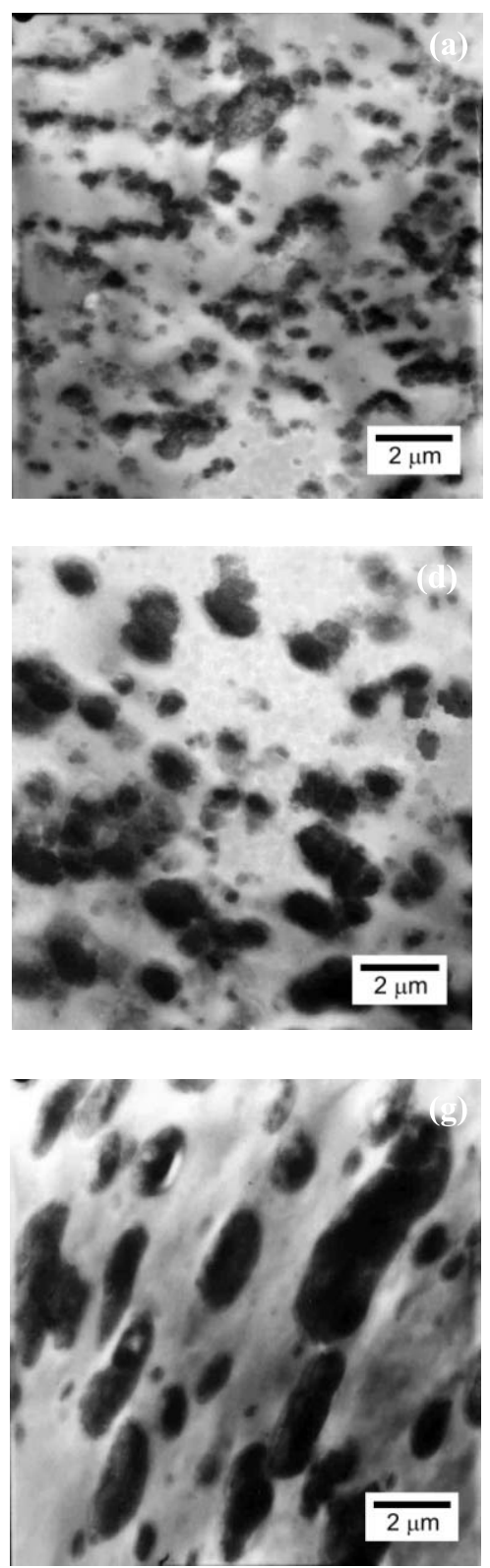
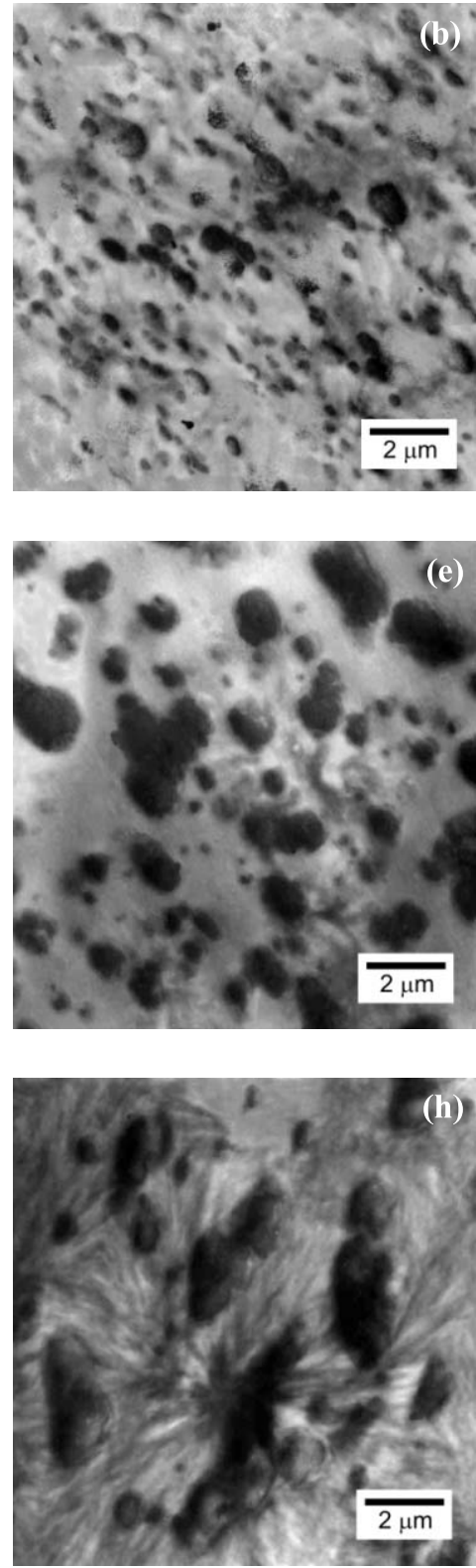
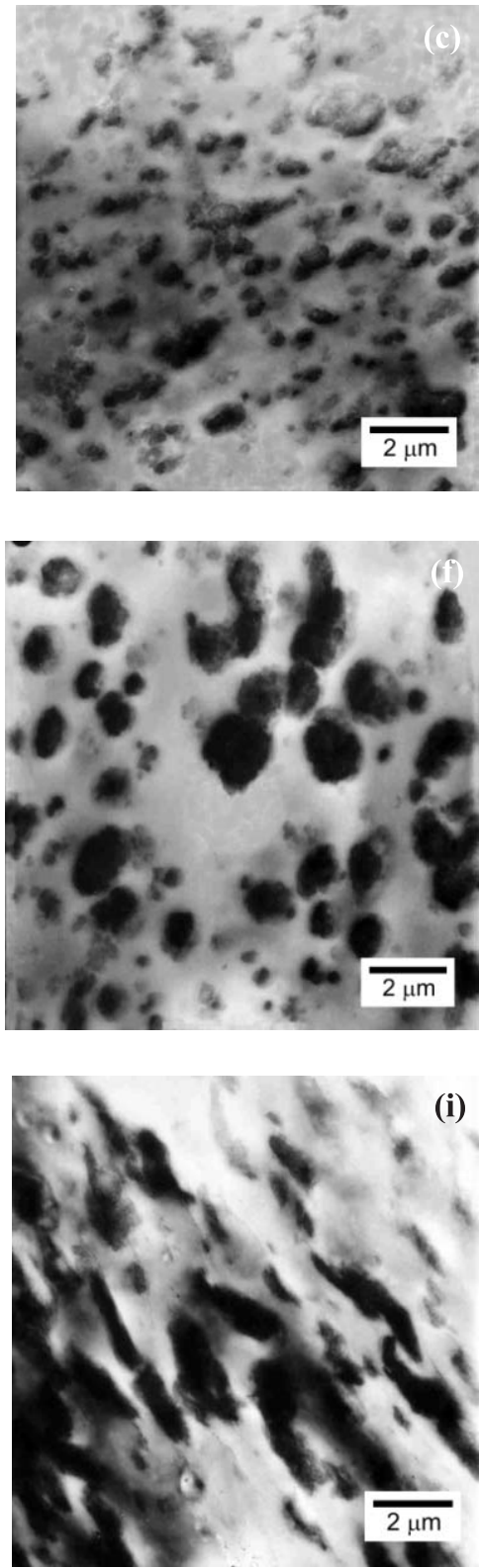

Figure 9. Fotomicrografias das blendas PA6/AES/MMA-MA1 (66,5/28,5/5) coletadas em diferentes posições na extrusora: (a) válvula B; (b) válvula C; (c) válvula $\mathrm{D}$; (d) válvula $\mathrm{E}$; (e) válvula F; (f) válvula $\mathrm{H}$; (g) válvula I; (h) saída da extrusora $\mathrm{M}$ e (i) corpo de prova injetado.

tículas, como pode ser observado nas Figuras $8 \mathrm{~h}$ e $9 \mathrm{~h}$, respectivamente.

A adição de compatibilizantes em blendas imiscíveis tende a reduzir a tensão interfacial entre os componentes devido à formação do copolímero na interface e assim produz uma estabilização contra a coalescência em diferentes estágios do processamento. A adição do compatibilizante MMA-MA1 resultou em diminuição no tamanho das partículas, mas não conseguiu promover a estabilização contra a coalescência das fases durante o processamento. Este aumento no tamanho das partículas pode ter sido intensificado pela presença dos vários elementos mistura ao longo do comprimento da rosca. Adição de um teor mais elevado do copolímero MMA-MA1 pode ser necessário a fim de promover a estabilização contra coalescência da fase dispersa AES neste sistema.
A moldagem por injeção também causou alterações na morfologia das fases, o que pode ser verificado comparando a morfologia das amostras extrudadas (Figuras $7 \mathrm{~h}, 8 \mathrm{~h}$ e $9 \mathrm{~h}$ ) com a morfologia das amostras injetadas (Figuras 7i, 8i e 9i). Percebe-se que com a adição do copolímero MMA-MA foi obtida maior eficiência na redução do tamanho das partículas da fase dispersa e isto está relacionado com a melhoria das propriedades mecânicas, porém, não foi obtido estabilidade da morfologia de fases ao longo dos processos de extrusão e de moldagem por injeção.

\section{Conclusões}

A incorporação do copolímero AES na matriz de PA6 não foi efetivo na melhoria da resistência ao impacto à tempera- 
tura ambiente, sendo que a temperatura de transição dútilfrágil obtida para a blenda polimérica PA6/AES foi superior à da PA6 pura. Por sua vez, a adição do copolímero MMAGMA10 ao sistema PA6/AES, também não promoveu melhoria nas propriedades de resistência ao impacto e na temperatura de transição dúctil-frágil. Estes resultados se correlacionam com a morfologia das fases observadas através de microscopia eletrônica de transmissão para estes sistemas, onde o AES não apresenta uma boa dispersão na matriz de PA6. Por outro lado, o copolímero MMA-MA1 é um compatibilizante bastante efetivo para o sistema PA6/AES, pois apresenta um efeito positivo sobre as propriedades de impacto e tração do sistema. A adição de somente $5 \%$ em peso do copolímero MMA-MA1 aumentou a resistência ao impacto à temperatura ambiente e reduziu a temperatura de transição dútil-frágil em aproximadamente $40^{\circ} \mathrm{C}$.

A evolução da morfologia das fases durante o processo de extrusão e em amostras injetadas é bastante distinta para os sistemas compatibilizados e não compatibilizados. Para as blendas ternárias PA6/AES/MMA-MA1 observa-se uma redução significante no tamanho das partículas dispersas logo no início do processo de extrusão, imediatamente após a fusão dos materiais. $\mathrm{O}$ mesmo fenômeno não foi observado para a blenda binária não compatibilizada e para a blenda ternária com MMA-GMA10. Foi possível também verificar a influência da matriz em promover a ruptura das partículas na saída da extrusora. O menor tamanho das partículas dispersa foi obtido em amostras injetadas para a blenda compatibilizada com MMA-MA1, sendo isto correlacionado com a melhora nas propriedades mecânicas. O copolímero MMA-MA1 foi um compatibilizante bastante efetivo para o sistema PA6/AES devido à reatividade dos grupos anidrido maleico do copolímero preferencialmente com os grupos finais amina da poliamida- 6 .

\section{Agradecimentos}

À BASF e Uniroyal Chemical pelo fornecimento dos materiais; à FAPESP e PRONEX/FINEP/CNPq pelo suporte financeiro.

\section{Referências Bibliográficas}

1. Zimmerman, J. - Encyclopedia of Polymer Science and Engineering - Polyamides, John Wiley \& Sons, 11, pp. 315-381 (1985)

2. Kohan, M. I. - Nylon Plastic, New York: John Wiley \& Sons (1973)

3. Lu, M. ; Keskkula H. and Paul, D. R. - Toughening of nylon6 with grafted rubber impact modifiers, J. Appl. Pol. Sci., 58, pp. 1175 (1995)

4. Royaltuf modified EPDM for tougher engineering thermoplastics and other rubber and plastics application, Uniroyal Chemical (1992)
5. Wefer, J. M. - Properties and structure of polyesterpolycarbonate blends containing grafted EPDM, SPEANTEC Tech. Papers (1990)

6. Kawanishi; K.; Tsuchikawa, S. and Kimura, S. - A study on polyamide 4,6/AES alloy, Composite Interfaces, 3, pp. 185 (1995)

7. Majundar; B.; Keskkula, H.; Paul, D. R. and Harvey, N. G. Control of the morphology of polyamide/styreneacrylonitrile copolymer blends via reactive compatibilizers, Polymer, 35, pp. 4263 (1994)

8. Majundar, B.; Paul, D. R. and Oshinski, A. J. - Evolution of morphology in compatibilized vs uncompatibilized polyamide blends, Polymer, 38, pp. 1787 (1997)

9. Jeon, H. K. and Kim, J. K. - Morphological development with time for immiscible polymer blends with in situ compatibilizers under controlled shear conditions, Polymer, 39, pp. 6227 (1998)

10. Dedecker, K. and Groeninckx, G. - Reactive compatibilization of $A /(B / C)$ polymer blends. Part 1. Investigation of the phase morphology development and stabilization, Polymer, 39, pp. 4985 (1998)

11. Cartier, H. and Hu, G. H. - Morphology development of in situ copatibilized semicrystalline polymer blends in a co-rotating twin-screw extruder, Pol. Eng. and Sci., 39, pp. 996 (1999)

12. Shearer, G. and Tzoganakis, C. - Analysis of mixing during melt-melt blending in twin screw extruders using reactive polymer tracers, Pol. Eng. and Sci., 39, pp. 1584 (1999)

13. Machado, A. V.; Covas, J. A. and Van Duin, M. - Chemical and morphological evolution of PA-6/EPM/EPM- $-M A$ in a twin screw extruder, J. Pol. Sci: Part A: Pol. Chem., 37, pp. 1311 (1999)

14. Machado, A. V.; Covas, J. A. and Van Duin, M. - Evolution of morphology and chemical conversion along the screw in a corotating twin-screw extruder, J. Appl. Pol. Sci., 71, pp. 135 (1999)

15. Lee, J. K. and Han, C. D. - Evolution of polymer blend morphology during compounding in a twin-screw extruder, Polymer, 41, pp. 1799 (2000)

16. Potente, H.; Bastian, M.; Bergemann, K.; Senge, M.; Scheel, G. and Winkelmann, T. - Morphology of polymer blends in the melting section of co-rotating twin screw extruders, Pol. Eng. and Sci., 41, pp. 222 (2001)

17. Machado, A. V.; Covas, J. A.; Wallet, M. and Van Duin, M. - Effect of composition and processing conditions on the chemical and morphological evolution of PA-6/ EPM/EPM-g-MA in a corotating twin-screw extruder, J. Appl. Pol. Sci, 80, pp. 1535 (2001) 
18. Araújo, E. M. - Tenacificação da Poliamida-6 com ABS por meio da Técnica de Compatibilização in situ com o uso de Copolímeros Acrílicos, PhD Thesis, Department of Materials Engineering, Universidade Federal de São Carlos (2001)

19. Kudva, R. A.; Keskkula, H. and Paul, D. R. - Compatibilization of nylon 6/ABS blends using glycidyl methacrylate/methyl methacrylate copolymers, Polymer, 39, pp. 2447 (1998)

20. Bassani, A.; Hage, E. and Pessan, L. A. - Effects of composition, processing conditions and reactive compatibilization on the mechanical properties of PA6/ AES blends, PPS 18, Guimarães, Portugal (2002)

21. Litauszki, B.; Schmidt-Naake, G.; Kressler, J. and Kammer, H. W. - Miscibility of poly(methyl methacrylate-co-maleic anhydride) with styrene- acrylonitrile and methyl methacrylate-styrene copolymers, Polymer Communications, 30, pp. 359 (1989)

22. Majundar, B.; Keskkula, H. and Paul, D. R. - Mechanical properties and morphology of nylon-6/acrylonitrilebutadiene-styrene blends compatibilized with imidized acrylic polymers, Polymer, 35, pp. 5453 (1994)

23. Paul, D. R.; Bucknall, C. B. Polymer Blends. New York: John Wiley \& Sons, v. 1 e 2, 2000.

24. Duin, M. van; Aussems, M.; Borggreve, R. J. M. Graft formation, chain scission, morphology and rheology in blends of polyamide- 6 and 6,6 with maleic anhydride containing polymers. In press.

Enviado: 15/06/04 Reenviado: 29/03/05 Aprovado: 01/04/05 